\title{
Self-Assembly of Dendrimer-Encapsulated Nanoparticle Arrays Using 2-D Microbial S-Layer Protein Biotemplates
}

Sonny. S. Mark, ${ }^{* \dagger}$ Magnus Bergkvist, ${ }^{\dagger}$ Xin Yang, ${ }^{\S}$ Esther R. Angert, ${ }^{\dagger}$ and Carl. A. Batt ${ }^{\S}$

${ }^{\dagger}$ Department of Microbiology, ${ }^{\dagger}$ and ${ }^{\S}$ Department of Food Science at Cornell University, Ithaca, New York 14853, "Present Address: Nanobiotechnology Center at Cornell University, Ithaca, New York 14853.

Supporting Information 


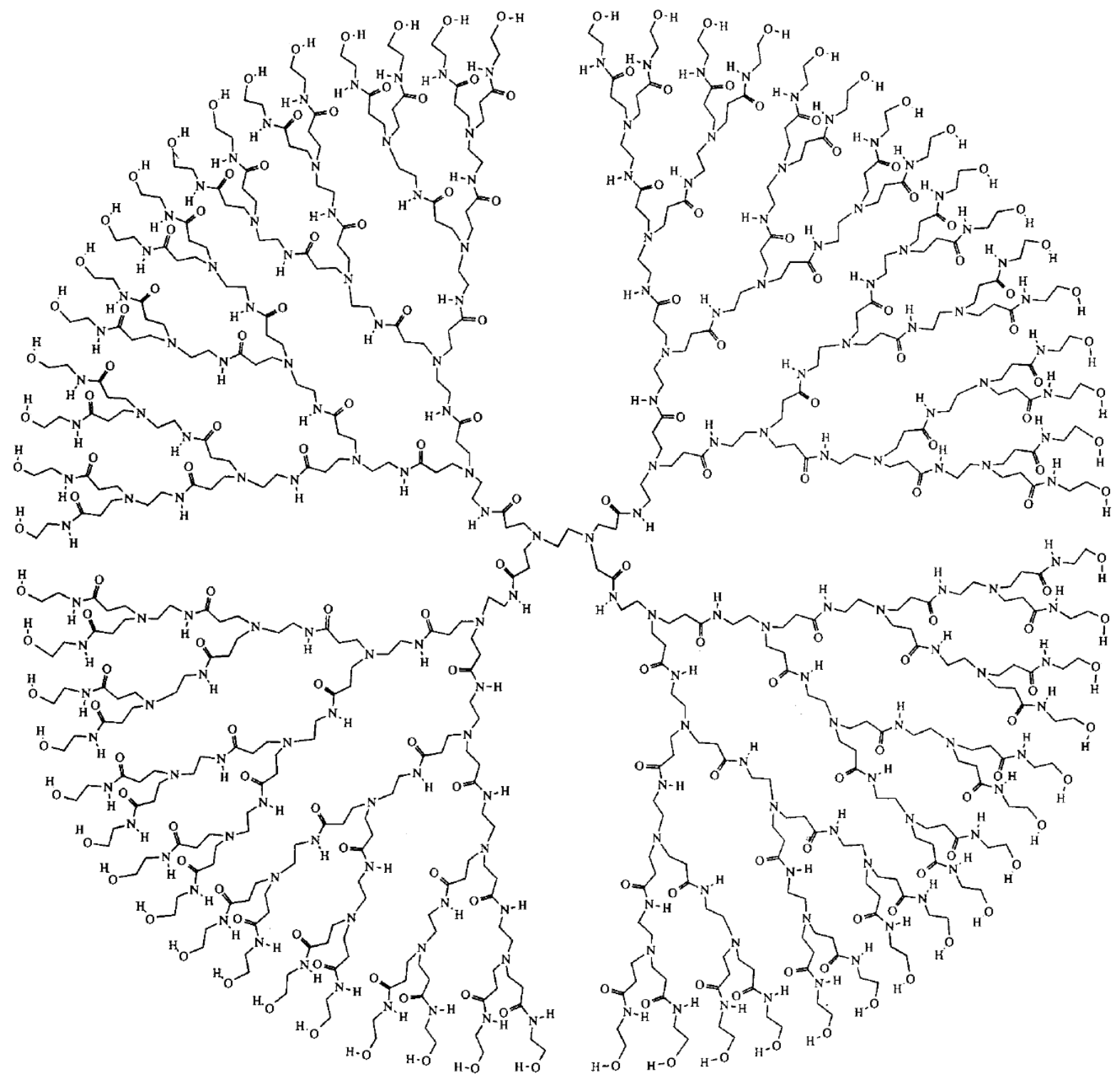

Core:

$-\mathrm{NCH}_{2} \mathrm{CH}_{2} \mathrm{~N}-$

Repetitive Unit: $\quad-\mathrm{N}\left(\mathrm{CH}_{2} \mathrm{CH}_{2} \mathrm{CONHCH}_{2} \mathrm{CH}_{2}\right)_{2}-$

Terminal Group: $\quad$-OH

Figure S1. Chemical structure of a generation 4 poly(amidoamine) (PAMAM) dendrimer bearing 64 surface hydroxyl groups (G4 PAMAM-OH). 
A

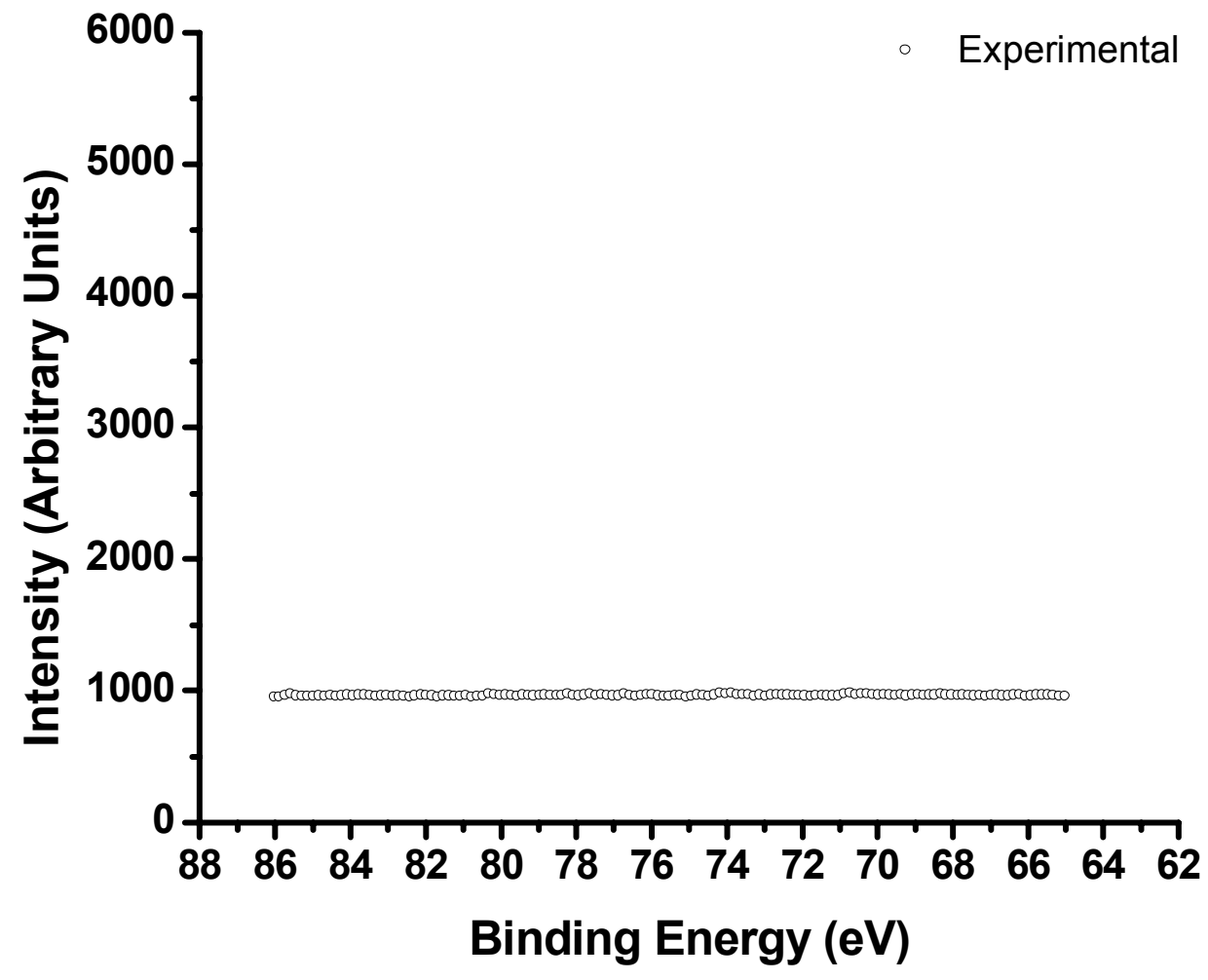

B

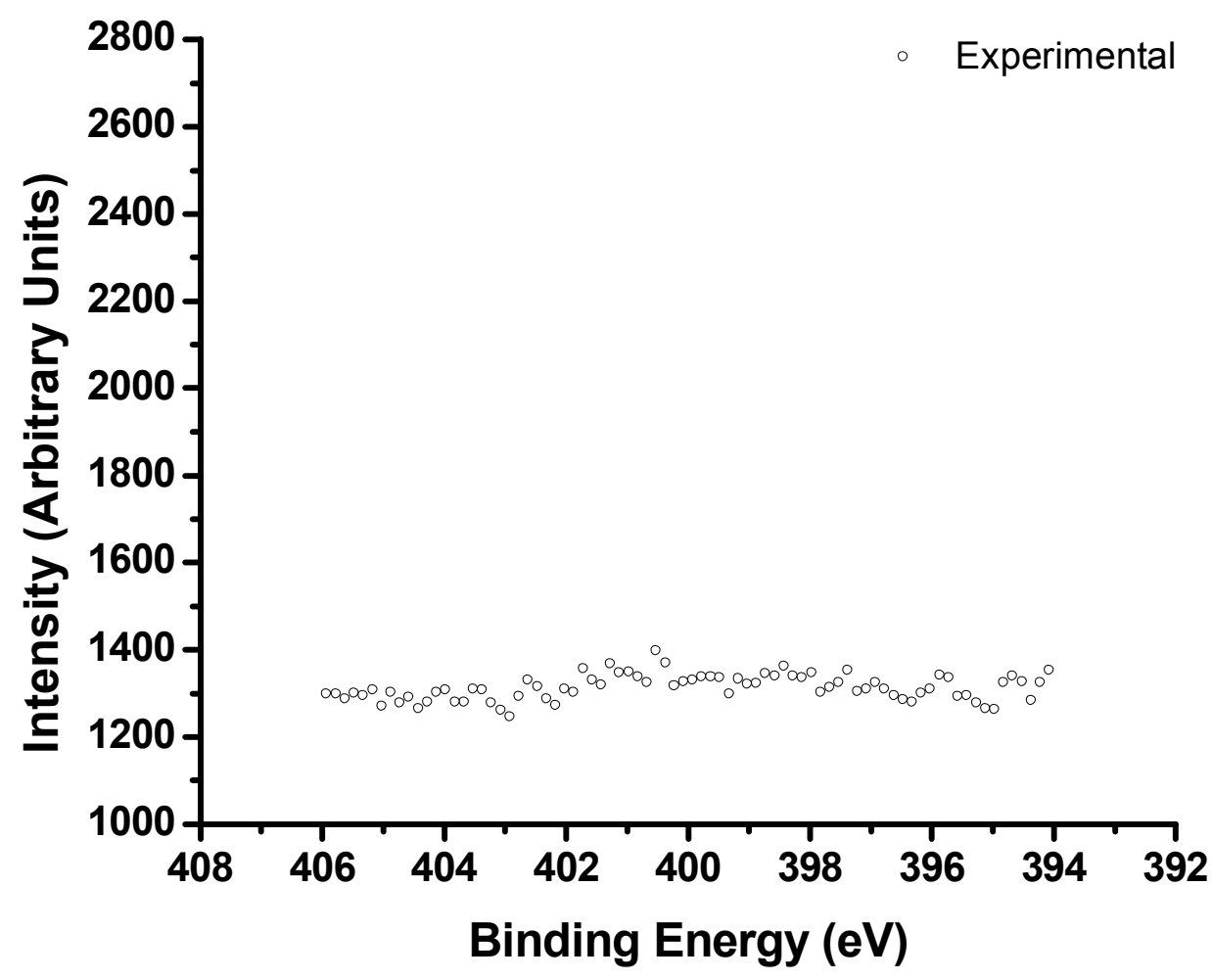

Figure S2. XPS spectra obtained for a blank silicon (100) wafer substrate.

A. Pt $4 f$ region of the highresolution XPS spectrum. B. N $1 s$ region of the high-resolution XPS spectrum. 
(a)
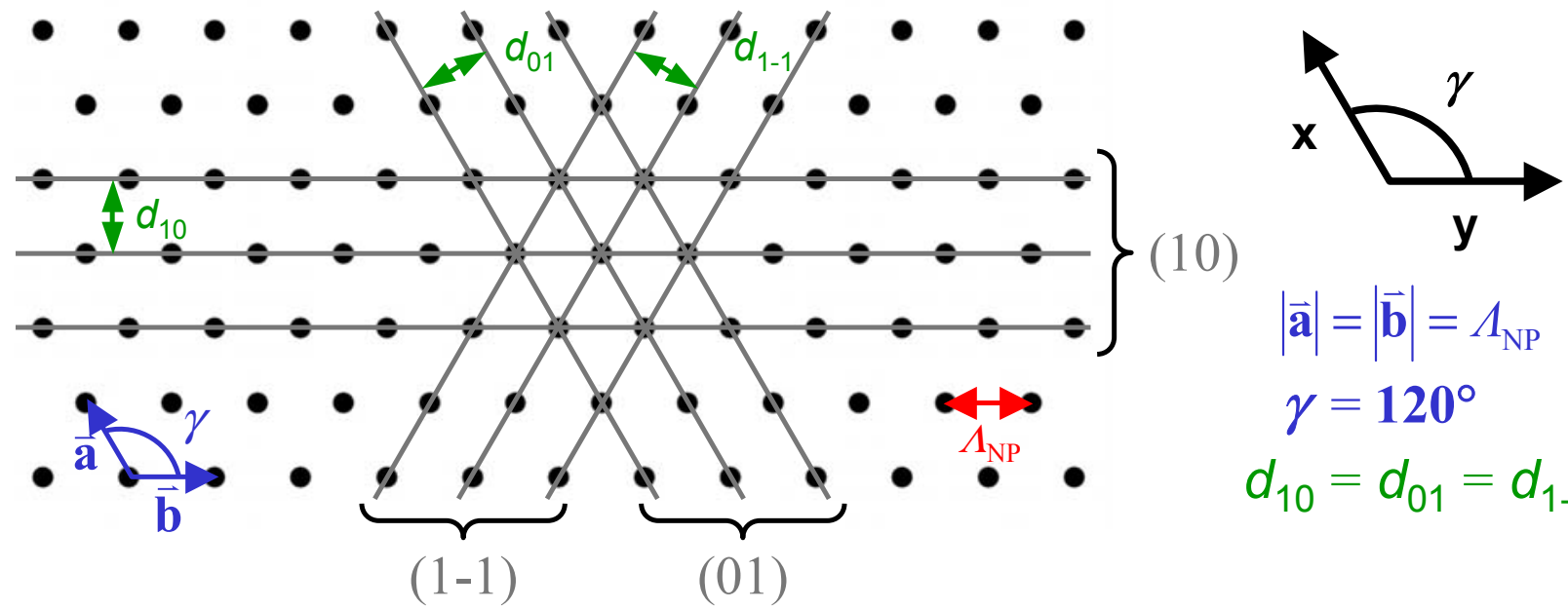

$$
\begin{aligned}
|\overrightarrow{\mathbf{a}}| & =|\overrightarrow{\mathbf{b}}|=\Lambda_{\mathrm{NP}} \\
\boldsymbol{\gamma} & =\mathbf{1 2 0}^{\circ} \\
d_{10} & =d_{01}=d_{1-1}
\end{aligned}
$$

(b)
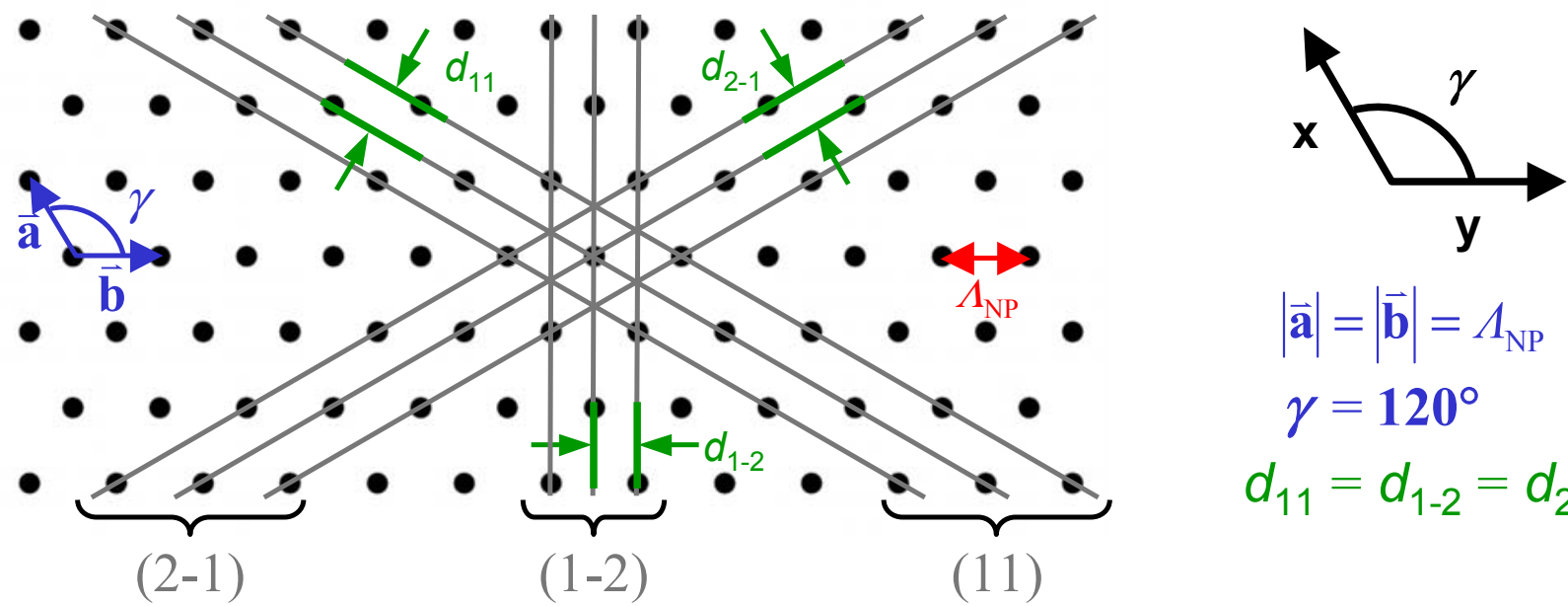

$$
\begin{aligned}
|\overrightarrow{\mathbf{a}}| & =|\overrightarrow{\mathbf{b}}|=\Lambda_{\mathrm{NP}} \\
\gamma & =\mathbf{1 2 0 ^ { \circ }} \\
d_{11} & =d_{1-2}=d_{2-1}
\end{aligned}
$$

(c)
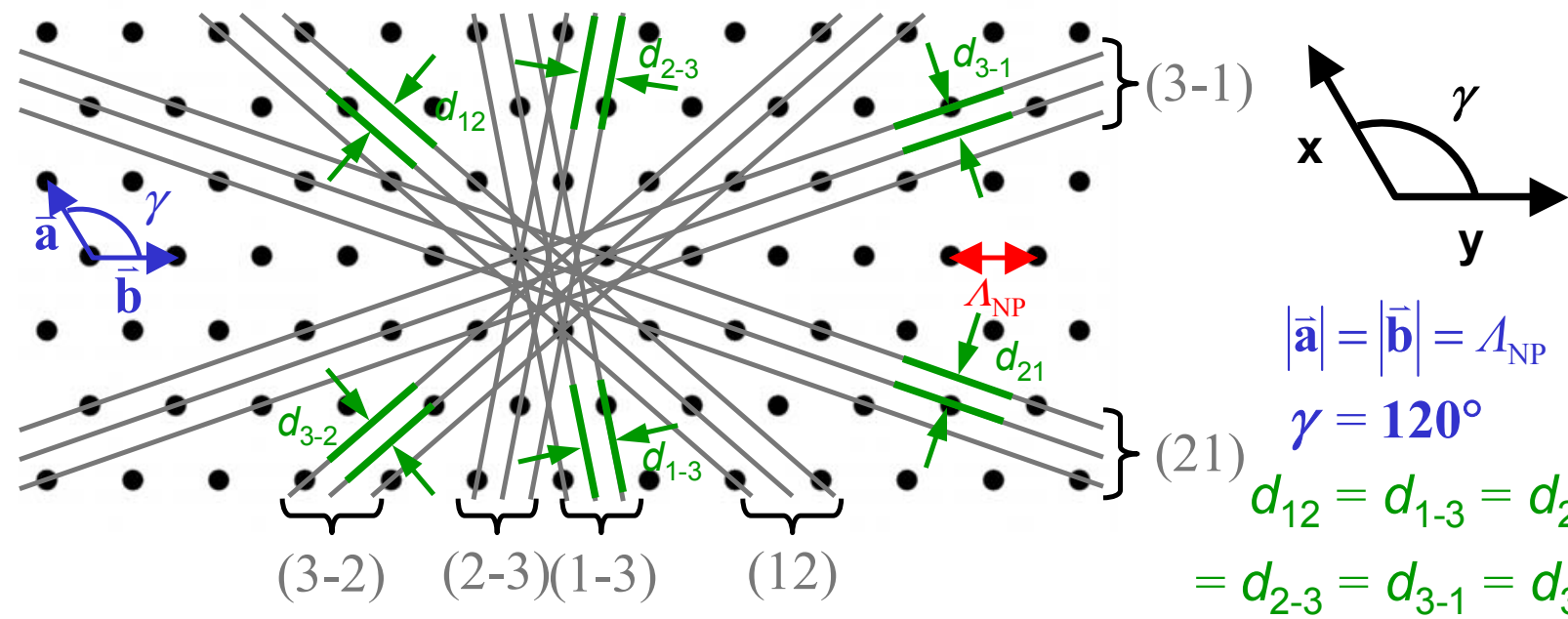

$$
\begin{aligned}
|\overrightarrow{\mathbf{a}}| & =|\overrightarrow{\mathbf{b}}|=\Lambda_{\mathrm{NP}} \\
\gamma & =\mathbf{1 2 0}^{\circ} \\
d_{12} & =d_{1-3}=d_{21} \\
=d_{2-3} & =d_{3-1}=d_{3-2}
\end{aligned}
$$

Figure S3. Schematic illustration of the major lattice orientations in an ideal two-dimensional hexagonal array structure. $\overrightarrow{\mathbf{a}}$ and $\overrightarrow{\mathbf{b}}$ are the primitive hexagonal unit cell vectors. ( $h k$ ) is the two-index specification of a set of parallel lines in the 2-D array, analogous to the Miller indices $(h k l)$ of a set of planes in a 3-D crystal. $d_{h k}$ ("lattice spacing") is the orthogonal distance between two lattice lines of a given set. $\Lambda_{\mathrm{NP}}$ ("lattice constant") is the interparticle nearest-neighbor (center-to-center) distance. (a) The set of lattice lines with $d_{\mathrm{hk}}=\Lambda_{\mathrm{NP}}$ $\left(\cos \left(30^{\circ}\right)\right)$. (b) The set of lattice lines with $d_{\mathrm{hk}}=\Lambda_{\mathrm{NP}} / 2$. (c) The set of lattice lines with $d_{\mathrm{hk}}=\Lambda_{\mathrm{NP}}\left(\cos \left(30^{\circ}\right) / \sqrt{ } 7\right)$. 